\title{
Association between atherogenic dyslipidemia and recurrent stroke risk in patients with different subtypes of ischemic stroke
}

\author{
Lu Zhao'†, Ruihao Wang ${ }^{1,2}+$, Bo Song1', Song Tan³, Yuan Gao', Hui Fang ${ }^{1}$, Jie Lu', and \\ Yuming $\mathrm{Xu}^{1 *}$
}

Background The association between atherogenic dyslipidemia and stroke recurrence remains unclear, and may be influenced by different subtypes of ischemic stroke.

Aims We aimed to investigate whether atherogenic dyslipidemia contributed to stroke recurrence in ischemic stroke patients and in those with certain subtypes of ischemic stroke. Methods We conducted a prospective hospital-based study enrolling patients with acute ischemic stroke. Atherogenic dyslipidemia was defined as high-density lipoprotein cholesterol $<\mathbf{4 0} \mathrm{mg} / \mathrm{dl}$ and triglycerides $\geq \mathbf{2 0 0} \mathrm{mg} / \mathrm{dl}$. Ischemic stroke subtypes were classified according to the Trial of Org 10172 in Acute Stroke Treatment criteria. The patients were followed up at 3, 6, 12 and 24 months after stroke onset. The association between atherogenic dyslipidemia and stroke recurrence was analyzed by using multivariable Cox regression model.

Results In the 510 ischemic stroke patients, 64 patients (12.5\%) had atherogenic dyslipidemia, and 66 patients (12.9\%) experienced stroke recurrence events within 24 months. KaplanMeier analysis revealed that stroke recurrence rate was significantly higher in patients with atherogenic dyslipidemia than those without in all the stroke patients $(20.3 \%$ vs. $11.9 \%$; $P=0.048$ ), and more evident in those of large-artery atherosclerosis subtype $(31.0 \%$ vs. $14.1 \% ; P=0.014)$, but not in the other subtypes. Multivariable Cox regression analysis revealed that atherogenic dyslipidemia was associated with higher stroke recurrence risk among stroke patients of large-artery atherosclerosis subtype (hazard ratio, 2.79; $95 \%$ confidence interval, 1.24-6.28), but not significant in all the stroke patients (hazard ratio, 1.69; $95 \%$ confidence interval, 0.85-3.37).

Conclusions Atherogenic dyslipidemia is associated with higher risk of stroke recurrence in ischemic stroke patients. Such association might be more pronounced in large-artery atherosclerosis subtype and needs further investigation to establish such relationship.

Correspondence: Yuming Xu*, Department of Neurology, the First Affiliated Hospital of Zhengzhou University, No. 1 East Jianshe Road, Zhengzhou, Henan 450052, China.

E-mail: xuyuming@zzu.edu.cn

${ }^{1}$ Department of Neurology, The First Affiliated Hospital of Zhengzhou University, Zhengzhou, Henan, China

${ }^{2}$ Department of Neurology, University of Erlangen-Nuremberg, Erlangen, Germany

${ }^{3}$ Department of Neurology, Sichuan Academy of Medical Sciences and Sichuan Provincial People's Hospital, Chengdu, Sichuan, China

${ }^{4}$ Department of Medical Statistics, College of Public Health, Zhengzhou University, Zhengzhou, Henan, China

Received: 1 September 2014; Accepted: 24 November 2014; Published online 28 Apirl 2015

$\dagger$ These authors contributed equally to this manuscript.

Conflict of interest: None declared.

Funding: This study was funded by the Youth Foundation of the First Affiliated Hospital of Zhengzhou University and Science and Technology Agency of Henan Province.

DOI: $10.1111 /$ ijs. 12471
Key words: atherogenic dyslipidemia, ischemic stroke, recurrent event

Survivors of acute ischemic stroke (IS) are at high risk of stroke recurrence $(1,2)$. Unfortunately, despite best medical treatment, there is still a high residual risk of cardiovascular diseases $(3,4)$. Atherogenic dyslipidemia (AD), which is defined as low highdensity lipoprotein cholesterol (HDL-C) and high triglycerides, may be one explanation for the high residual risk of stroke (5).

$\mathrm{AD}$ is common in patients with established cardiovascular disease, type 2 diabetes mellitus and metabolic syndrome (5). Patients with $\mathrm{AD}$ presented a wide range of abnormalities, including increased plasma concentrations of remnants, denser LDL, more atherogenic HDL particles, and insulin resistant status (6). Thus, AD may characterize stroke patients with higher stroke recurrence risk.

However, data on the association between $\mathrm{AD}$ and stroke recurrence in patients with stroke or transient ischemic attack (TIA) are limited $(7,8)$. And IS is a heterogenous disease with variable pathogenesis (9). AD therefore may not contribute to subsequent strokes in patients with different stroke subtypes equally. To our knowledge, there is no data on the influence of $\mathrm{AD}$ on stroke recurrence in patients with different subtypes of IS. Thus we carried out this prospective hospital-based study to explore the prevalence of $\mathrm{AD}$ and its contribution to stroke recurrence among a cohort of patients with acute IS.

\section{Patients and methods}

Patients included in this study were from the database of the Henan Province Stroke Registry which prospectively collected data on consecutive stroke patients within 14 days after stroke onset in the Department of Neurology of the First Affiliated Hospital of Zhengzhou University (Henan, China) and followed up for long periods (10). This study was approved by the Ethics Committee of the First Affiliated Hospital of Zhengzhou University, and all patients or their designated relatives signed written informed consent. The present study involved patients enrolled in the registry between January 2010 and July 2011.

\section{Baseline data collection}

Baseline data of the patients were recorded in paper-based case report forms. Acute IS was diagnosed according to the World Health Organization Criteria (11). The demographics included age, gender, body weight, height and body mass index (BMI). The stroke risk factors included history of stroke (defined as medical record confirmed history of ischemic, hemorrhagic stroke or subarachnoid hemorrhage), hypertension (defined as history of hypertension and newly diagnosed hypertension), 
diabetes mellitus (including history of diabetes mellitus and newly diagnosed diabetes), history of coronary heart disease (including myocardial infarction and angina pectoris), atrial fibrillation (confirmed by at least one electrocardiogram or present during hospitalization). The personal history mainly consisted of current or previous smoking, and moderate or heavy drinking (defined as $\geq$ two standard alcohol consumption per day). The severity of neurologic impairment was evaluated according to the National Institutes of Health Stroke Scale (NIHSS) within 24 hours after admission by trained research investigators through direct interview with the patient at bedside (12). Stroke subtypes were classified according to the Trial of ORG 10172 in Acute Stroke Treatment (TOAST) criteria by two trained study neurologists. IS subtypes included large-artery atherosclerosis (LAA), cardioembolism (CE), small-artery occlusion (SAO), stroke of other determined causes (OC) and stroke of undetermined causes (UC) (13). We combined the stroke of OC and UC types as 'other subtypes' group. Blood samples were collected in fasting conditions for evaluation of the lipid profiles, fasting plasma glucose (FPG) and other routine tests, the lipid profiles included total cholesterol (TC), triglycerides (TG), high-density lipoprotein cholesterol (HDL-C) and low-density lipoprotein cholesterol (LDL-C). Atherogenic dyslipidemia was defined as low HDL-C and high triglycerides in consistent with previous studies $(6,7,14,15)$. The cutoff values were selected at HDL-C $<40 \mathrm{mg} / \mathrm{dl}$ $(1.04 \mathrm{mmol} / \mathrm{l})$ and triglyceride $\geq 200 \mathrm{mg} / \mathrm{dl}(2 \cdot 26 \mathrm{mmol} / \mathrm{l})$ in both men and women (16).

\section{Follow-up of stroke recurrence}

At 3, 6, 12 and 24 months after stroke onset, all patients or their relatives were followed through telephone and asked whether the patients had new symptoms and experienced re-hospitalization with a diagnosis of ischemic or hemorrhagic stroke. A recurrent stroke was defined as a newly diagnosed stroke presenting as a new neurological deficit or a deterioration of the previous deficit (11), not considered to be because of edema, hemorrhagic transformation, or concurrent illness. Hospitals that admitted patients with recurrent strokes were contacted to verify the diagnosis. Follow-up was performed by trained and qualified telephone interviews who were not involved in the registry and blinded to the baseline data.

\section{Statistical analysis}

For descriptive analysis, data were expressed as mean ( \pm standard deviation) for normally distributed continuous variables and median (interquartile range) for non-normally distributed continuous variables, and counts (percentages) for categorical variables. Bivariate comparisons were conducted using the $\chi^{2}$ test for categorical variables, and Student $t$ test or Mann-Whitney test for normally distributed or non-normally distributed continuous variables. Kaplan-Meier analysis was used to compare the stroke recurrence rate between patients with and without atherogenic dyslipidemia. The association between atherogenic dyslipidemia and stroke recurrence was analyzed by multivariable Cox regression models by adjusting for confounders which were associated with $\mathrm{AD}$ in the bivariate comparisions and/or stroke recurrence in univariable Cox regression models $(P<0 \cdot 1)$. Unadjusted and adjusted hazard ratios (HRs) and 95\% confidence intervals (95\% CIs) were calculated. Statistical tests were conducted, with a 2 -tailed $\alpha$-level of $0 \cdot 05$ considered significant. Data were analyzed using SPSS version 17.0 (SPSS Inc., Chicago, IL).

\section{Results}

A total of 566 patients with acute IS were consecutively registered. 551 patients had complete baseline information. Among them, 41 patients $(7 \cdot 4 \%)$ were lost to follow-up, leaving 510 patients for the final analysis. There was no significant difference of baseline clini$\mathrm{cal}$ and laboratory characteristics between the patients with complete follow-up data $(n=510)$ and those lost to follow-up $(n=41)$ (Online-only Data Supplemental Table S1). In the 510 patients with follow-up data, the average age was $60 \cdot 69 \pm 13.90$ years, and 319 cases $(62.5 \%)$ were women. According to the TOAST classification, there were 234 cases (45.9\%) with LAA, 192 cases $(37 \cdot 6 \%)$ with SAO, 23 cases $(4 \cdot 5 \%)$ with CE, 16 cases $(3 \cdot 1 \%)$ with other determined causes, and 45 cases $(8 \cdot 8 \%)$ of undetermined causes.

$\mathrm{AD}$ was observed in 64 patients $(12 \cdot 5 \%)$. Table 1 showed characteristics in patients with and without $\mathrm{AD}$. Compared with patients without $\mathrm{AD}$, patients with $\mathrm{AD}$ had significantly higher BMI (25.97 \pm 3.35 vs. $24 \cdot 43 \pm 4.55 ; P=0 \cdot 012)$, TC $(5 \cdot 07 \pm 1 \cdot 21$ vs. $4.64 \pm 1 \cdot 18 ; P=0.006)$ and FPG $(7 \cdot 64 \pm 3 \cdot 13$ vs. $6 \cdot 13 \pm 2 \cdot 81$; $P<0 \cdot 001)$, and were more likely to have diabetes mellitus $(40 \cdot 6 \%$ vs. $22.2 \%, P=0.001)$. The remaining baseline data did not differ between the two groups. The prevalence of $\mathrm{AD}$ did not differ between different TOAST subtypes of stroke ( $P=0.754$; Table 2$)$.

During the 2-year-follow-up period, stroke recurrence events happened in 66 patients (12.9\%). Kaplan-Meier analysis revealed that the stroke recurrence rate in patients with $\mathrm{AD}$ was significantly higher in all the stroke patients $(20 \cdot 3 \%$ vs. $11.9 \%$; $P=0 \cdot 048)$ and stroke patients of LAA subtype (31.0\% vs. $14 \cdot 1 \%$; $P=0.014$ ) (Table 2; Fig. 1), insignificantly lower in the SAO subtype $(4 \cdot 3 \%$ vs. $10 \cdot 1 \% ; P=0 \cdot 393)$, and insignificantly higher in CE subtype ( $50 \cdot 0 \%$ vs. $14 \cdot 3 \%$; $P=0 \cdot 268$ ) or other subtypes (consisting of other determined causes and undetermined causes) (20.0\% vs. $7 \cdot 8 \% ; P=0 \cdot 198)$ (Table 2). Unadjusted Cox proportional regression analysis showed that $\mathrm{AD}$ was borderlinely associated with higher stroke recurrence risk in all the stroke patients (HR, 1.82; 95\% CI, 0.99-3.35; $P=0.052$ ) and was significantly associated with an increased stroke recurrence risk among stroke patients of LAA subtype (HR, 2.47; 95\% CI, 1·17-5·23; $P=0 \cdot 018$ ) (Table 3). Online-only Data Supplemental Table S2 displayed potential variables associated with stroke recurrence. After adjusting for age, hypertension, diabetes mellitus, NIHSS score at admission, BMI, and total cholesterol, the association was attenuated in all the stroke patients (HR, 1.69; 95\% CI, 0.85-3.37; $P=0 \cdot 135)$ but still significant in stroke patients of LAA subtype (HR, 2.79; 95\% CI, 1·24-6.28; $P=0 \cdot 013$ ) (Table 3; Fig. 2).

\section{Discussion}

In this prospective hospital-based study, we found that the prevalence of $\mathrm{AD}$ among patients with acute IS was $12 \cdot 5 \%$, patients 
Table 1 Patient characteristics in ischemic stroke patients with and without atherogenic dyslipidemia

\begin{tabular}{|c|c|c|c|}
\hline \multirow[b]{2}{*}{ Variable } & \multicolumn{2}{|c|}{ Atherogenic dyslipidemia* } & \multirow[b]{2}{*}{$P$ value } \\
\hline & Yes $(N=64)$ & No $(N=446)$ & \\
\hline \multicolumn{4}{|l|}{ Demographics } \\
\hline Age, y & $58 \cdot 77 \pm 13 \cdot 71$ & $60 \cdot 97 \pm 13 \cdot 92$ & 0.236 \\
\hline Women & 39 (60.9\%) & $280(62 \cdot 8 \%)$ & 0.776 \\
\hline NIHSS Score at Admission & $4(1-7)$ & $4(2-8)$ & 0.459 \\
\hline $\mathrm{BMI}, \mathrm{kg} / \mathrm{m}^{2}$ & $25 \cdot 97 \pm 3 \cdot 35$ & $24.43 \pm 4.55$ & 0.012 \\
\hline \multicolumn{4}{|l|}{ Risk Factors } \\
\hline Hypertension & $43(67 \cdot 2 \%)$ & $257(57 \cdot 6 \%)$ & $0 \cdot 146$ \\
\hline DM & $26(40 \cdot 6 \%)$ & $99(22 \cdot 2 \%)$ & 0.001 \\
\hline Coronary Heart Disease & $5(7 \cdot 8 \%)$ & $57(12 \cdot 8 \%)$ & 0.255 \\
\hline Atrial Fibrillation & $3(4 \cdot 7 \%)$ & $17(3 \cdot 8 \%)$ & 0.716 \\
\hline Previous Stroke History & $28(43 \cdot 8 \%)$ & $116(26 \cdot 0 \%)$ & 0.533 \\
\hline Current or Previous Smoking & $23(35 \cdot 9 \%)$ & $163(36 \cdot 5 \%)$ & 0.925 \\
\hline Moderate or Heavy Drinking & $16(25 \cdot 0 \%)$ & $115(25 \cdot 8 \%)$ & 0.893 \\
\hline \multicolumn{4}{|l|}{ Blood Biochemical Tests } \\
\hline Hemoglobin, g/l & $139 \cdot 76 \pm 24 \cdot 33$ & $134.73 \pm 18.66$ & $0 \cdot 120$ \\
\hline Fibrinogen, $g / l$ & $3.06 \pm 0.88$ & $3 \cdot 34 \pm 1 \cdot 89$ & 0.257 \\
\hline Homocysteine, $\mathrm{mmol} / \mathrm{l}$ & $21 \cdot 10 \pm 11 \cdot 51$ & $20 \cdot 50 \pm 10 \cdot 96$ & 0.718 \\
\hline $\mathrm{TC}, \mathrm{mmol} / \mathrm{l}$ & $5 \cdot 07 \pm 1 \cdot 21$ & $4 \cdot 64 \pm 1 \cdot 18$ & 0.006 \\
\hline LDL-C, $\mathrm{mmol} / / \mathrm{l}$ & $3.00 \pm 0.87$ & $3.03 \pm 0.96$ & $0 \cdot 814$ \\
\hline $\mathrm{FPG}, \mathrm{mmol} / \mathrm{l}$ & $7 \cdot 64 \pm 3 \cdot 13$ & $6 \cdot 13 \pm 2 \cdot 81$ & $<0.001$ \\
\hline TOAST Classification & & & 0.754 \\
\hline LAA Subtype & $29(45 \cdot 3 \%)$ & $205(46.0 \%)$ & \\
\hline SAO Subtype & $23(35 \cdot 9 \%)$ & $169(37.9 \%)$ & \\
\hline CE Subtype & $2(3 \cdot 1 \%)$ & $21(4 \cdot 7 \%)$ & \\
\hline Other Subtypes ${ }^{\dagger}$ & $10(15 \cdot 6 \%)$ & $51(11 \cdot 4 \%)$ & \\
\hline \multicolumn{4}{|c|}{ Secondary Prevention Medications Application } \\
\hline Antithrombotic Drugs & $46(71.9 \%)$ & $309(69 \cdot 3 \%)$ & 0.590 \\
\hline Lipid-lowering Drugs & $20(31 \cdot 3 \%)$ & $164(36 \cdot 8 \%)$ & 0.394 \\
\hline Antihypertensive Drugs & $35(54 \cdot 7 \%)$ & $211(47 \cdot 3 \%)$ & 0.241 \\
\hline Hypoglycemic Drugs & $18(28 \cdot 1 \%)$ & $87(19 \cdot 5 \%)$ & $0 \cdot 104$ \\
\hline
\end{tabular}

*Defines as HDL-C $<40 \mathrm{mg} / \mathrm{dl}(1.04 \mathrm{mmol} / \mathrm{l})$ and triglyceride $\geq 200 \mathrm{mg} / \mathrm{dl}(2.26 \mathrm{mmol} / \mathrm{l})$.

${ }^{\dagger}$ Other Subtypes consisted of stroke subtypes other determined causes and undetermined causes.

NIHSS indicates National Institutes of Health Stroke Scale; BMI, Body Mass Index; DM, Diabetes mellitus; TC, total cholesterol; LDL-C, low-density lipoprotein cholesterol; FPG, fasting plasma glucose; TOAST, Trial of ORG 10172 in Acute Stroke Treatment; LAA, large-artery atherosclerosis; SAO, small-artery occlusion; CE indicated cardiac embolism.

Table 2 Prevalence of atherogenic dyslipidemia and its association with stroke recurrence among different subtypes of stroke

\begin{tabular}{|c|c|c|c|c|c|}
\hline \multirow[b]{2}{*}{ Stroke subtypes } & \multirow[b]{2}{*}{ AD prevalence* } & \multicolumn{3}{|c|}{ Stroke recurrence rate } & \multirow[b]{2}{*}{$P$ value } \\
\hline & & All & $\mathrm{AD}(+)$ & $A D(-)$ & \\
\hline All Subtypes $(n=510)$ & $64(12 \cdot 5 \%)$ & $66(12 \cdot 9 \%)$ & $13(20 \cdot 3 \%)$ & $53(11.9 \%)$ & 0.048 \\
\hline LAA Subtype $(n=234)$ & $29(12 \cdot 4 \%)$ & $38(16 \cdot 2 \%)$ & $9(31 \cdot 0 \%)$ & $29(14 \cdot 1 \%)$ & 0.014 \\
\hline SAO Subtype $(n=192)$ & $23(12 \cdot 0 \%)$ & $18(9 \cdot 4 \%)$ & $1(4 \cdot 3 \%)$ & $17(10 \cdot 1 \%)$ & 0.393 \\
\hline CE Subtype $(n=23)$ & $2(8.7 \%)$ & $4(17 \cdot 4 \%)$ & $1(50 \cdot 0 \%)$ & $3(14 \cdot 3 \%)$ & 0.268 \\
\hline Other Subtypes $(n=61)^{\dagger}$ & $10(16 \cdot 4 \%)$ & $6(9 \cdot 8 \%)$ & $2(20 \%)$ & $4(7 \cdot 8 \%)$ & 0.198 \\
\hline
\end{tabular}

AD defines as HDL-C $<40 \mathrm{mg} / \mathrm{dl}(1.04 \mathrm{mmol} / \mathrm{l})$ and triglyceride $\geq 200 \mathrm{mg} / \mathrm{dl}(2.26 \mathrm{mmol} / \mathrm{l})$.

*No statistically significant difference according to Chi-Square test, $P=0.754$.

${ }^{+}$Other Subtypes consisted of stroke subtypes other determined causes and undetermined causes.

AD indicates atherogenic dyslipidemia; LAA, large-artery atherosclerosis; SAO, small-artery occlusion; CE, cardiac embolism.

with $\mathrm{AD}$ had a significantly higher stroke recurrence risk than those without, and the association between $\mathrm{AD}$ and increased stroke recurrence risk appeared more pronounced among stroke patients of LAA subtype.
Our findings in the prevalence and clinical characteristics of $\mathrm{AD}$ were consistent with the previous studies $(7,8)$. The prevalence of $\mathrm{AD}$ in SOS-TIA study was $11 \cdot 1 \%$ among TIA patients with a cerebral ischemic lesion (7), and a recently published post 

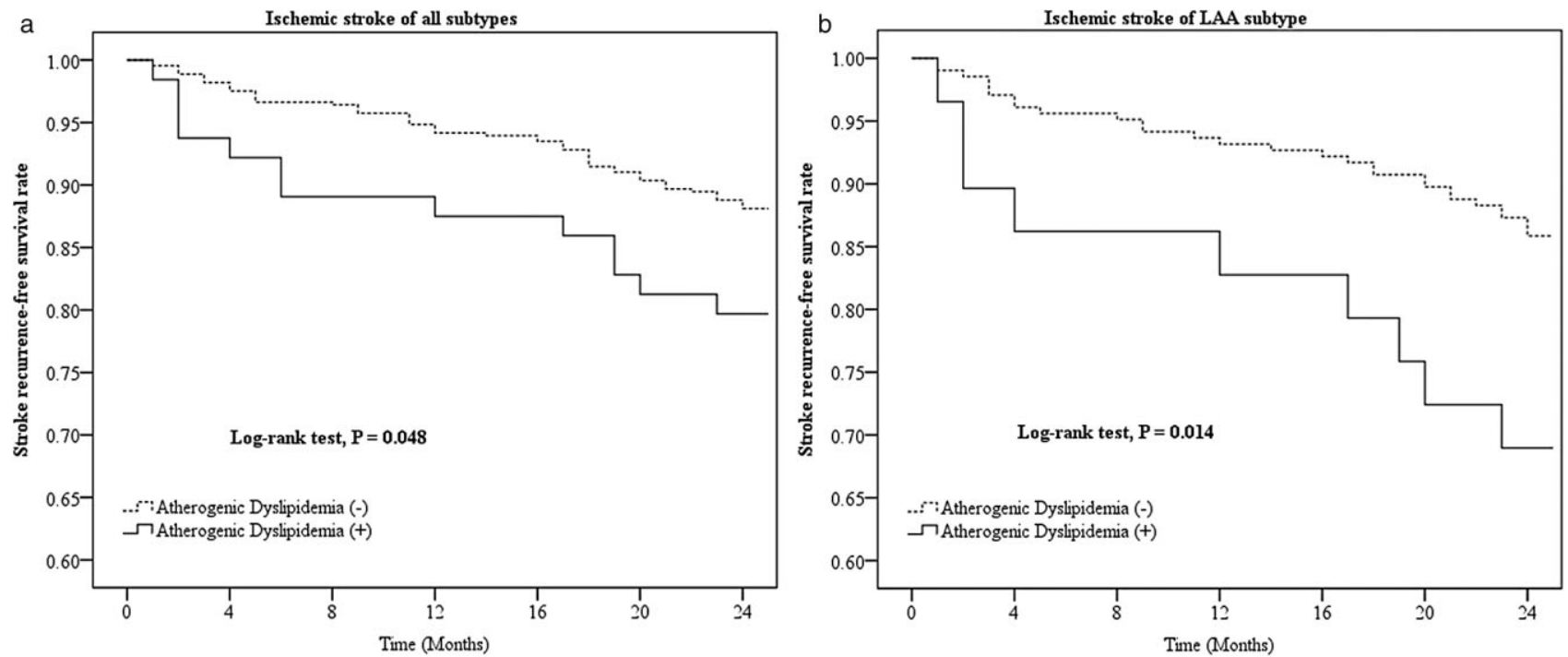

Fig. 1 Kaplan-Meier curves of stroke recurrence rate in patients with ischemic stroke of all subtypes (a) and those of large-artery atherosclerosis subtype (b) with and without AD.

Table 3 Cox proportional regression analysis of atherogenic dyslipidemia and stroke recurrence

\begin{tabular}{|c|c|c|c|c|c|c|}
\hline & \multicolumn{2}{|c|}{ All subtypes of stroke } & \multicolumn{2}{|l|}{ LAA subtype } & \multicolumn{2}{|c|}{ Non-LAA subtypes* } \\
\hline & Unadjusted & Adjusted & Unadjusted & Adjusted & Unadjusted & Adjusted \\
\hline \multicolumn{7}{|l|}{$A D$} \\
\hline HR & $1 \cdot 82$ & 1.69 & $2 \cdot 47$ & $2 \cdot 79$ & $1 \cdot 18$ & 0.49 \\
\hline $95 \% \mathrm{Cl}$ & $0 \cdot 99-3 \cdot 35$ & $0.85-3 \cdot 37$ & $1 \cdot 17-5 \cdot 23$ & $1 \cdot 24-6 \cdot 28$ & $0 \cdot 41-3 \cdot 39$ & $0 \cdot 11-2 \cdot 19$ \\
\hline$P$ Value & 0.052 & 0.135 & 0.018 & 0.013 & 0.762 & 0.353 \\
\hline \multicolumn{7}{|l|}{ Age } \\
\hline $\mathrm{HR}$ & 1.02 & $1 \cdot 02$ & $1 \cdot 02$ & $1 \cdot 02$ & 1.04 & $1 \cdot 04$ \\
\hline $95 \% \mathrm{Cl}$ & $1.00-1.04$ & $1.00-1.04$ & $1.00-1.04$ & $0.99-1.04$ & $1.01-1.07$ & $1.00-1.07$ \\
\hline$P$ Value & 0.016 & 0.049 & 0.202 & 0.298 & 0.023 & 0.037 \\
\hline \multicolumn{7}{|c|}{ Hypertension } \\
\hline $\mathrm{HR}$ & $2 \cdot 11$ & $2 \cdot 18$ & $2 \cdot 38$ & $2 \cdot 59$ & $1 \cdot 81$ & 1.64 \\
\hline $95 \% \mathrm{Cl}$ & $1 \cdot 21-3 \cdot 66$ & $1 \cdot 21-3 \cdot 94$ & $1 \cdot 13-5.03$ & $1 \cdot 14-5 \cdot 88$ & $0 \cdot 80-4 \cdot 12$ & $0.68-3.94$ \\
\hline$P$ Value & 0.008 & 0.010 & 0.023 & 0.023 & $0 \cdot 155$ & 0.272 \\
\hline \multicolumn{7}{|c|}{ Diabetes Mellitus } \\
\hline $\mathrm{HR}$ & 1.83 & $1 \cdot 54$ & 1.63 & $1 \cdot 37$ & $2 \cdot 24$ & $2 \cdot 60$ \\
\hline $95 \% \mathrm{Cl}$ & $1 \cdot 11-3 \cdot 02$ & $0.89-2 \cdot 66$ & $0 \cdot 82-3 \cdot 23$ & $0.67-2 \cdot 82$ & $1.06-4.73$ & $1 \cdot 10-6 \cdot 14$ \\
\hline$P$ Value & 0.018 & 0.124 & 0.162 & $0 \cdot 391$ & 0.035 & 0.029 \\
\hline \multicolumn{7}{|c|}{ NIHSS score } \\
\hline $\mathrm{HR}$ & 1.04 & 1.04 & 1.00 & 1.00 & 1.07 & 1.08 \\
\hline $95 \% \mathrm{Cl}$ & $1.00-1.08$ & $1.00-1.08$ & $0.94-1.06$ & $0.93-1.06$ & $1 \cdot 02-1 \cdot 13$ & $1 \cdot 02-1 \cdot 13$ \\
\hline$P$ Value & 0.065 & 0.062 & 0.864 & 0.887 & 0.005 & 0.005 \\
\hline \multicolumn{7}{|l|}{ BMl } \\
\hline $\mathrm{HR}$ & 0.97 & 0.96 & 1.00 & 0.97 & 0.94 & 0.89 \\
\hline $95 \% \mathrm{Cl}$ & $0.93-1.02$ & $0.91-1.00$ & $0.94-1 \cdot 1$ & $0.91-1.03$ & $0.87-1.03$ & $0.79-1.01$ \\
\hline$P$ Value & $0 \cdot 262$ & 0.066 & 0.899 & 0.309 & 0.168 & 0.069 \\
\hline \multicolumn{7}{|c|}{ Total Cholesterol } \\
\hline $\mathrm{HR}$ & $1 \cdot 04$ & 0.96 & 1.08 & 0.89 & 1.02 & $1 \cdot 16$ \\
\hline $95 \% \mathrm{Cl}$ & $0.86-1 \cdot 26$ & $0 \cdot 76-1 \cdot 20$ & $0.83-1.39$ & $0 \cdot 67-1 \cdot 18$ & $0.76-1.38$ & $0.77-1.76$ \\
\hline$P$ Value & 0.674 & 0.709 & 0.587 & 0.414 & 0.888 & 0.474 \\
\hline
\end{tabular}

*Non-LAA subtypes included stroke subtypes of small-artery occlusion, cardiac embolism, other determined caused and undetermined causes. LAA indicates large-artery atherosclerosis; HR, hazard ratio; Cl, confidence ratio; NIHSS, National Institutes of Health Stroke Scale; BMI, Body Mass Index. 

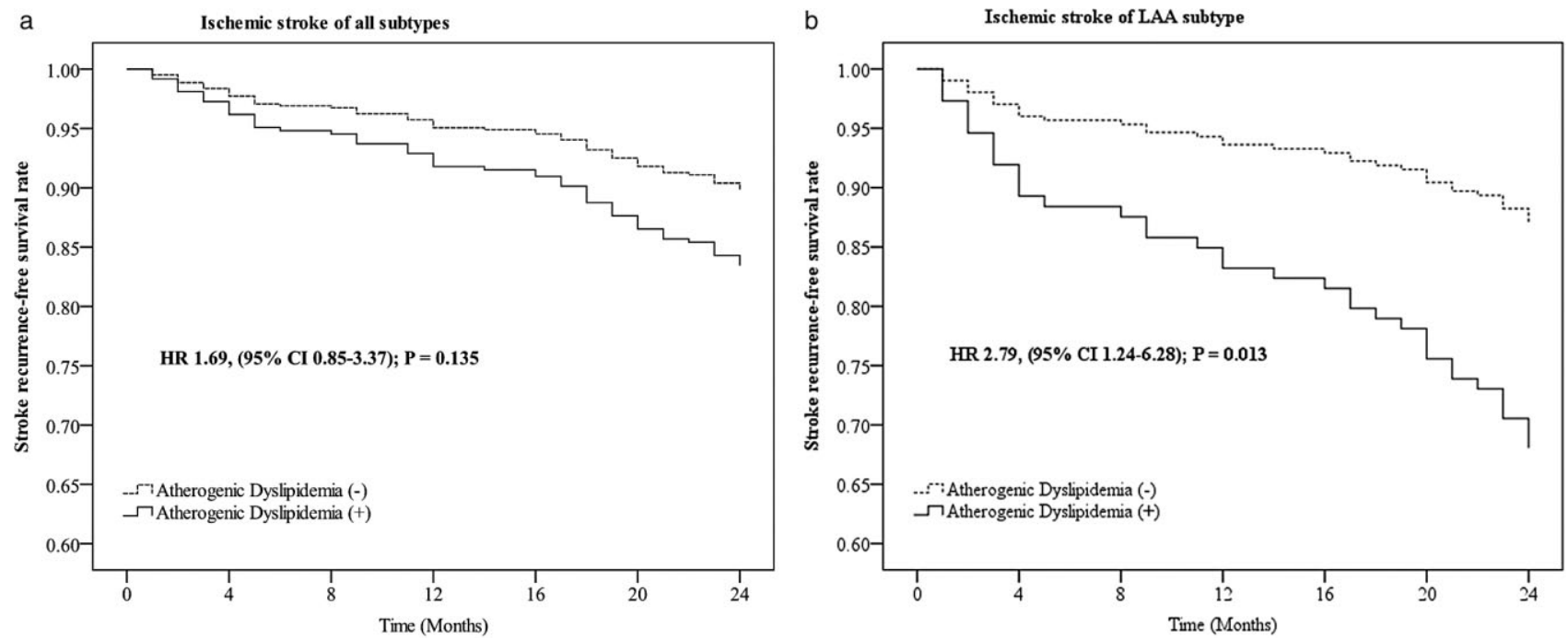

Fig. 2 Adjusted survival curves derived from Cox proportional model of stroke recurrence rate in patients with ischemic stroke of all subtypes (a) and those of large-artery atherosclerosis subtype (b) with and without AD. Age, hypertension, diabetes mellitus, NIHSS score at admission, BMI, and total cholesterol were adjusted.

hoc study of two large clinical trials found that approximately 1 in 10 patients with TIA or stroke had AD (8). We found that stroke patients with $\mathrm{AD}$ were more likely to have diabetes, higher BMI and FPG value, and this might be explained by insulin resistance, which was considered to play an important role in the pathogenic basis of atherogenic dyslipidemia (15).

Our data showed that stroke patients with AD had significantly increased stroke recurrence risk than those without AD. Some published studies have addressed the prognostic value of the individual components of $\mathrm{AD}$ in patients with cerebrovascular diseases $(17,18)$. A post hoc analysis of the Stroke Prevention by Aggressive Reduction in Cholesterol Levels (SPARCL) trail found that in patients with recent stroke or TIA, lower baseline HDL-C predicted the risk of recurrent stroke, and high triglyceride level was associated with major cardiovascular event (18). Fukuoka Stroke Registry study revealed low HDL-C level was an independent risk factor for a stroke recurrence within one year after onset (17). To our knowledge, only two researches addressed the impact of $\mathrm{AD}$ on the stroke recurrence in patients with stroke or TIA. The deleterious role of $\mathrm{AD}$ in cerebrovascular diseases was first studied in the SOS-TIA study, which found that AD might be associated with intracranial artery stenosis and higher risk of early recurrent stroke among patients with TIA (7). A recently published post hoc study of SPARCL and PERFORM (Prevention of Cerebrovascular and Cardiovascular Events of Ischemic Origin With Terutroban in Patients With a History of Ischemic Stroke or Transient Ischemic Attack) trail found that among subjects with stroke or TIA while receiving statin and otherwise best medical therapy, those with $\mathrm{AD}$ had a higher residual cardiovascular risk than those without AD (8).

The most important finding of this study was that $\mathrm{AD}$ was independently associated with increased recurrent stroke risk among stroke patients of LAA subtype. The post hoc study of SPARCL trail showed the association between AD and major cardiovascular events in patients with IS or TIA became insignificant after multivariable adjustment (HR 1.24; 95\% CI, 0.93-1.65), and in the study of PERFORM trial, the association was attenuated after multivariable adjustment (HR 1.23; 95\% CI, 1.03-1.48). However, the analyses were not performed in the different stroke subtypes (8). We hypothesize that $\mathrm{AD}$ may not equally contribute to subsequent stroke events among stroke patients of LAA and SAO subtypes equally. IS is a heterogeneous disease, the etiology of different TOAST subtypes varies (19), and the pathogenesis of stroke recurrence also differs (20). Kumral et al found a significant association between baseline dyslipidemia and stroke recurrence in the large-artery disease subtype, but not in the other stroke subtypes (21). Mounting evidence support the strong association between lipid disorder and large artery atherosclerosis stroke (2224). Warfarin-Asprin Symptomatic Intracranial Disease (WASID) study revealed that lipid disorder has the most strongest association of the severity of the severity of intracranial large artery stenosis (23). Bang OY et al found that elevated levels of serum triglycerides and non-high-density lipoprotein, but not lowdensity lipoprotein (LDL), were associated with large atherosclerotic stroke (22). Chinese Intracranial Atherosclerosis(CICAS) study revealed that low HDL-C level is associated with the development of intracranial artery stenosis in Chinese patients with acute IS (24). In contrast, the pathogenesis of small-artery occlusion stroke has not been fully clarified, but is considered to be due to nonatherosclerotic arteriopathy, which is mainly caused by hypertension and diabetes (25). Atherosclerosis Risk in Communities (ARIC) study found that lacunar infarcts caused by the occlusion of small arteries were associated with diabetes or LDL cholesterol (26). These findings support that AD may cardinally contribute to stroke recurrence in stroke patients of LAA, which could explain our results. Our study indicates stroke patients of LAA subtype may benefit from the combination of fibrate and statin, which was not yet supported by the current evidence from the Action to Control Cardiovascular Risk in Diabetes (ACCORD) study conducted in the stroke patients which were not classified by stroke etiology, however the subgroup analysis of the ACCORD study showed individuals with $\mathrm{AD}$ seemed to 
benefit from the combination (27). A further meta-analysis also supported that fibrate treatment directed at markers of AD substantially reduced subsequent vascular event risk (14).

Our study had limitations. First, it was a hospital-based study conducted only in one hospital, the sample size was small, and hospital selective bias was inevitable (28). As the sample sizes in CE subtype and other subtypes of IS were small, type II error (false negative association between $\mathrm{AD}$ and stroke recurrence) might happen due to a lack of statistical power, we still could not determine the relationship between $\mathrm{AD}$ and stroke recurrence in CE subtype and other subtypes or conclude whether AD contributed to the recurrence of different stroke subtypes. Second, as Peduzzi et al suggested that the number of outcome events per variable $(\mathrm{EPV})=10$ to be most prudent in a proportional hazards regression analysis (29), the value of EPV in our multivariable Cox proportional regression analysis seemed not high enough to exclude overfitting (overestimation of the true effect), thus a confounding bias related to covariables might still exist despite adjustment. Third, lipid profiles during follow-up period which may influence prognosis were not examined. Fourth, the follow-up was obtained via telephone interview, which could bring inaccuracy comparing to a face-to-face clinic visit. Nevertheless, we tried to minimize such bias with telephone interviews conducted by trained and qualified telephone interviews, and hospitals that admitted patients with recurrent strokes were contacted to verify the diagnosis. Finally, although we classified the subtypes of initial IS, we could not classify the subtypes of recurrent stroke, as the follow-up information was obtained via telephone interview and was not available in the registry.

In conclusion, our study supports that atherogenic dyslipidemia is associated with higher risk of stroke recurrence in patients with ischemic stroke. Such association might be more pronounced in the LAA subtype, but requires further investigation to establish this relationship. Nevertheless, treatment measures to stroke patients with $\mathrm{AD}$, especially those of LAA subtype, might help reduce stroke recurrence rate.

\section{Acknowledgements}

The authors would like to thank all the patients and their relatives who participated in this study. We also thank Dr. Juan Gui (from Tongji Hospital, Huazhong University of Science and Technology, China) and Dr. Xinwu Cui (from Caritas hospital, Bad Mergentheim, Germany) for their assistance in writing the manuscript.

\section{References}

1 Donnan GA, Fisher M, Macleod M, Davis SM. Stroke. Lancet 2008; 371:1612-23.

2 Davis SM, Donnan GA. Clinical practice. Secondary prevention after ischemic stroke or transient ischemic attack. N Engl J Med 2012; 366:1914-22.

3 Hackam DG, Spence JD. Combining multiple approaches for the secondary prevention of vascular events after stroke: a quantitative modeling study. Stroke 2007; 38:1881-5.

4 Amarenco P, Labreuche J. Lipid management in the prevention of stroke: review and updated meta-analysis of statins for stroke prevention. Lancet Neurol 2009; 8:453-63.
5 Fruchart JC, Sacks F, Hermans MP et al. The Residual Risk Reduction Initiative: a call to action to reduce residual vascular risk in patients with dyslipidemia. Am J Cardiol 2008; 102:1K-34K.

6 Arca M, Montali A, Valiante S et al. Usefulness of atherogenic dyslipidemia for predicting cardiovascular risk in patients with angiographically defined coronary artery disease. Am J Cardiol 2007; 100:1511-6.

7 Sirimarco G, Deplanque D, Lavallee PC et al. Atherogenic dyslipidemia in patients with transient ischemic attack. Stroke 2011; 42:2131-7.

8 Sirimarco G, Labreuche J, Bruckert E et al. Atherogenic dyslipidemia and residual cardiovascular risk in statin-treated patients. Stroke 2014; 45:1429-36.

9 Saeed M. Editorial comment - unraveling the pagodian architecture of stroke as a complex disorder. Stroke 2004; 35:824-5.

10 Song B, Fang H, Zhao L et al. Validation of the ABCD3-I score to predict stroke risk after transient ischemic attack. Stroke 2013; 44:1244-8.

11 Stroke - 1989. Recommendations on stroke prevention, diagnosis, and therapy. Report of the WHO Task Force on Stroke and other Cerebrovascular Disorders. Stroke 1989; 20:1407-31.

12 Brott T, Adams HP Jr, Olinger CP et al. Measurements of acute cerebral infarction: a clinical examination scale. Stroke 1989; 20:86470.

13 Adams HP Jr, Bendixen BH, Kappelle LJ et al. Classification of subtype of acute ischemic stroke. Definitions for use in a multicenter clinical trial. TOAST. Trial of Org 10172 in Acute Stroke Treatment. Stroke 1993; 24:35-41.

14 Lee M, Saver JL, Towfighi A, Chow J, Ovbiagele B. Efficacy of fibrates for cardiovascular risk reduction in persons with atherogenic dyslipidemia: a meta-analysis. Atherosclerosis 2011; 217:492-8.

15 Cabre A, Babio N, Lazaro I et al. FABP4 predicts atherogenic dyslipidemia development. The PREDIMED study. Atherosclerosis 2012; 222:229-34.

16 National Cholesterol Education Program (NCEP) Expert Panel on Detection, Evaluation, and Treatment of High Blood Cholesterol in Adults (Adult Treatment Panel III). Third Report of the National Cholesterol Education Program (NCEP) Expert Panel on Detection, Evaluation, and Treatment of High Blood Cholesterol in Adults (Adult Treatment Panel III) final report. Circulation 2002; 106:3143-421.

17 Kuwashiro T, Sugimori H, Ago T, Kamouchi M, Kitazono T. Risk factors predisposing to stroke recurrence within one year of noncardioembolic stroke onset: the Fukuoka Stroke Registry. Cerebrovasc Dis 2012; 33:141-9.

18 Amarenco P, Goldstein LB, Callahan A 3rd et al. Baseline blood pressure, low- and high-density lipoproteins, and triglycerides and the risk of vascular events in the Stroke Prevention by Aggressive Reduction in Cholesterol Levels (SPARCL) trial. Atherosclerosis 2009; 204:515-20.

19 Hillen T, Coshall C, Tilling K, Rudd AG, McGovern R, Wolfe CD. Cause of stroke recurrence is multifactorial: patterns, risk factors, and outcomes of stroke recurrence in the South London Stroke Register. Stroke 2003; 34:1457-63.

20 Shin DH, Lee PH, Bang OY. Mechanisms of recurrence in subtypes of ischemic stroke: a hospital-based follow-up study. Arch Neurol 2005; 62:1232-7.

21 Kumral E, Evyapan D, Gokcay F, Karaman B, Orman M. Association of baseline dyslipidemia with stroke recurrence within five-years after ischemic stroke. Int J Stroke 2014; 9(Suppl. A100):119-26.

22 Bang OY, Saver JL, Liebeskind DS, Pineda S, Ovbiagele B. Association of serum lipid indices with large artery atherosclerotic stroke. Neurology 2008; 70:841-7.

23 Turan TN, Makki AA, Tsappidi S et al. Risk factors associated with severity and location of intracranial arterial stenosis. Stroke 2010; 41:1636-40.

24 Qian Y, Pu Y, Liu L et al. Low HDL-C level is associated with the development of intracranial artery stenosis: analysis from the Chinese IntraCranial AtheroSclerosis (CICAS) study. PLoS ONE 2013; 8:e64395. 
25 Jackson CA, Hutchison A, Dennis MS et al. Differing risk factor profiles of ischemic stroke subtypes: evidence for a distinct lacunar arteriopathy? Stroke 2010; 41:624-9.

26 Bezerra DC, Sharrett AR, Matsushita K et al. Risk factors for lacune subtypes in the Atherosclerosis Risk in Communities (ARIC) Study. Neurology 2012; 78:102-8.

27 Ginsberg HN, Elam MB, Lovato LC et al. Effects of combination lipid therapy in type 2 diabetes mellitus. N Engl J Med 2010; 362:1563-74.

28 Schulz UG, Rothwell PM. Differences in vascular risk factors between etiological subtypes of ischemic stroke: importance of populationbased studies. Stroke 2003; 34:2050-9.

29 Peduzzi P, Concato J, Feinstein AR, Holford TR. Importance of events per independent variable in proportional hazards regression analysis. II. Accuracy and precision of regression estimates. J Clin Epidemiol 1995; 48:1503-10.

\section{Supporting information}

Additional Supporting Information may be found in the online version of this article at the publisher's web-site:

Table S1. Comparisons of baseline characteristics of ischemic stroke patients with and lost to follow-up.

Table S2. Univariable Cox analysis for characteristics and recurrent stroke in 510 patients with ischemic stroke. 